$\mathrm{KN}-11$ Solving crystal structures with GSAS-II

Robert B. Von Dreele ${ }^{1}$

1. Advanced Photon Source, Argonne National Laboratory, Lemont, Il 60439 USA

email: vondreele@anl.gov

One of the goals in developing GSAS-II was to expand from the capabilities of the original General Structure Analysis System (GSAS) which largely encompassed just structure refinement and post refinement analysis. GSAS-II has been written almost entirely in Python loaded with graphics, GUI and mathematical packages (matplotlib, pyOpenGL, wx, numpy and scipy). Thus, GSAS-II has a fully developed modern GUI as well as extensive graphical display of data and results. However, the structure and operation of Python has required new approaches to many of the algorithms used in crystal structure analysis. The extensions beyond GSAS include image calibration/integration as well as peak fitting and unit cell indexing for powder data which are precursors for structure solution. Structure solution within GSAS-II begins with either Pawley extracted structure factors from powder data or those measured in a single crystal experiment. Both charge flipping and Monte Carlo-Simulated Annealing techniques are available; the former can be applied to $(3+1)$ incommensurate structures as well as conventional 3D structures. This talk will highlight the features of GSAS-II with particular focus on the structure solution facilities. Supported by US DOE/OS/BES under Contract No. DE-AC-02-06CH11357.

Keywords: charge flipping, GSAS-II, Rietveld refinement, Python

\section{KN-12 Time-resolved femtosecond crystallography opens a new era in Structure Biology \\ Petra Fromme}

1. Arizona State University, School of Molecular Sciences and center for Applied Structural Discovery Biodesign Institute

email: PFromme@asu.edu

Time-resolved Serial Femtosecond Crystallography (SFX) provides a novel concept for structure determination, where X-ray diffraction "snapshots" are collected from a fully hydrated stream of nanocrystals at room temperature, using femtosecond pulses from the world's first high energy X-ray free-electron laser, the Linac Coherent Light Source before the crystals are destroyed by the X-rays. The first proof of concept of serial femtosecond crystallography was achieved using Photosystem I, a larger membrane protein complex involved in Photosynthesis as a model system [1],[2]. In the time resolved SFX studies, the nano/microcrystals of Photosystem II [3] are fully active in water splitting and are cycled through the S-states by laser excitation "on the fly". Conformational changes of the $\mathrm{Mn}_{4} \mathrm{Ca}$ cluster and its protein environment were observed for the first time in the transition from the dark to the double excited state [4], however the resolution was limited to $5 \mathrm{~A}$ resolution. Very recently we have achieved a breakthrough, which cuts the "Gordian Knott" that will lead to a paradigm shift in X-ray crystallography. We have been able to show that "single molecule" continuous X-ray diffraction can be obtained by SFX from Photosystem II mircocrystals with minimal displacements of the PSII molecules by $0.8 \AA$, which is less than the bond length between two $\mathrm{C}$ atoms. These molecular continuous diffraction patterns were directly phased and the structure determined to a resolution way beyond the Bragg peaks [5]. The talk will also report on the development of compact femto and attosecond X-ray Sources at DESY (AXSIS) and at ASU (CXLS and CXFEL), which will provide unique new opportunities to study the ultrafast dynamics of reactions in photosynthesis with a combination of X-ray diffraction, X-ray spectroscopy and ultrafast optical spectroscopy. References: [1] Chapman,HN, Fromme,P, Barty, A. et al Nature 2011, 470, 73-77 [2] Fromme P., Spence JC. Curr Opin Struct Biol 2011, 21: 509-516 [3] Kupitz C, Grotjohann I, Conrad CE, Roy-Chowdhury S, Fromme R, Fromme ;P Philos Trans R Soc Lond B Biol Sci 2014, 369 [4] Kupitz, C, Basu, S, Grotjohann, I et al Nature 2014, 513, (7517), 261-5 [5] Ayyer, K. et al. Nature 2016 530, 202-206 


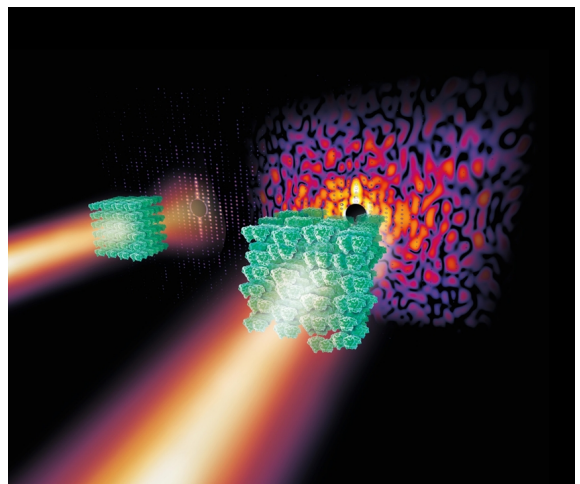

Figure 1. Artistic view of Bragg versus Continous Diffraction from crystals (Credit: DESY, Eberhard Reiman)

Keywords: Free Electron Laser, time-resolved femtosecond crystallography, Photosynthesis, Photosystem II, continous diffraction

\section{KN-13 Transition metal oxides: from structural complexity and topotactic oxygen diffusion pathways to energy applications \\ Werner Paulus ${ }^{1}$}

1. Institut Charles Gerhardt, ICGM-UMR 5253 CNRS Université de Montpellier, 34000 Montpellier, France

email: werner.paulus@univ-montp2.fr

Understanding oxygen diffusion in solid oxides at the atomic scale is an important issue for the development of technological devices such as solid oxide fuel cells (SOFC), as well as membrane based air separators, oxygen sensors and catalytic converters to transform e.g. $\mathrm{NO}$ or $\mathrm{CO}$ from exhaust emissions into $\mathrm{N}_{2}$ and $\mathrm{CO}_{2}^{\mathrm{x}}$ [1-2]. For SOFC e.g., the main drawback of long-term endurance is related to high operating temperatures of around $1000-1250{ }^{\circ} \mathrm{C}$, presenting a key challenge in terms of fatigue due to compositional and microstructural degradation of the respective electrode/membrane materials.

Regarding electrodes materials with mixed ionic and electronic transport properties, two structure types are known today to undergo oxygen intercalation reactions at ambient temperature: the Ruddlesden-Popper series, such as the $\mathrm{RE}_{2} \mathrm{MO}_{4+\mathrm{d}}(\mathrm{RE}=\mathrm{La}, \mathrm{Nd}, \mathrm{Pr} ; \mathrm{M}=\mathrm{Cu}, \mathrm{Ni}, \mathrm{Co})$ members, and the layered double perovskites labeled AA' $\mathrm{B}_{2} \mathrm{O}_{5+\mathrm{d}}$, including the brownmillerite structure, such as $\mathrm{SrMO}_{2.5}^{5+\mathrm{d}}(\mathrm{M}=\mathrm{Fe}, \mathrm{Co})$. We recently demonstrated that oxygen mobility at ambient temperature in brownmillerites is intimately related to low energy phonon modes, which constituted the first evidence of a phonon-assisted oxygen diffusion mechanism [3-4].

We discuss here complex oxygen ordering phenomena during oxygen uptake or release reactions in non-stoichiometric oxides with Brownmillerite and $\mathrm{K}_{2} \mathrm{NiF}_{4}$ type frameworks, essentially explored by in situ neutron scattering and synchrotron radiation. Oxygen doping frequently induces the generation of highly correlated systems for both, oxygen ordering but also for charge and orbital ordering, resulting in giant unit cells. We further discuss the interplay of highly correlated oxygen ordering and related changes in the lattice dynamics to better understand low temperature oxygen mobility, activated by a phonon-assisted diffusion mechanisms. Our studies allow to achieve a microscopic understanding of oxygen diffusion mechanism in solid oxides down to ambient temperature, which is equally important for the synthetic chemist to explore new, kinetically stabilized oxides not available by classical solid state methods [5].

1. Paulus W. et al. JACS 130, 47 (2008) 16080

2. Penkala B. et al. Cat Sci \& Tech, 5 (2015) 4839

3. Ceretti M et al. , J MatChem A, 3, 42 (2015) 21140

4. Perrichon A. et al. JPhysChem C119 (2015) 1557

5. Houchati I. et al. Chem. Mater. 24 (2012) 3811 